\title{
Comparative analysis of copy number variation detection methods and database construction
}

\author{
Asako Koike ${ }^{1 *}$, Nao Nishida ${ }^{2}$, Daiki Yamashita ${ }^{2}$, Katsushi Tokunaga ${ }^{2}$
}

\begin{abstract}
Background: Array-based detection of copy number variations (CNVs) is widely used for identifying disease-specific genetic variations. However, the accuracy of CNV detection is not sufficient and results differ depending on the detection programs used and their parameters. In this study, we evaluated five widely used CNV detection programs, Birdsuite (mainly consisting of the Birdseye and Canary modules), Birdseye (part of Birdsuite), PennCNV, CGHseg, and DNAcopy from the viewpoint of performance on the Affymetrix platform using HapMap data and other experimental data. Furthermore, we identified CNVs of 180 healthy Japanese individuals using parameters that showed the best performance in the HapMap data and investigated their characteristics.
\end{abstract}

Results: The results indicate that Hidden Markov model-based programs PennCNV and Birdseye (part of Birdsuite), or Birdsuite show better detection performance than other programs when the high reproducibility rates of the same individuals and the low Mendelian inconsistencies are considered. Furthermore, when rates of overlap with other experimental results were taken into account, Birdsuite showed the best performance from the view point of sensitivity but was expected to include many false negatives and some false positives. The results of 180 healthy Japanese demonstrate that the ratio containing repeat sequences, not only segmental repeats but also long interspersed nuclear element (LINE) sequences both in the start and end regions of the CNVs, is higher in CNVs that are commonly detected among multiple individuals than that in randomly selected regions, and the conservation score based on primates is lower in these regions than in randomly selected regions. Similar tendencies were observed in HapMap data and other experimental data.

Conclusions: Our results suggest that not only segmental repeats but also interspersed repeats, especially LINE sequences, are deeply involved in CNVs, particularly in common CNV formations.

The detected CNVs are stored in the CNV repository database newly constructed by the "Japanese integrated database project" for sharing data among researchers. http://gwas.lifesciencedb.jp/cgi-bin/cnvdb/cnv_top.cgi.

\section{Background}

Copy number variations (CNVs), duplications, and deletions of chromosomal segments longer than $1 \mathrm{~kb}$ are major structural variations in various organisms such as yeast, Drosophila, and humans and are believed to have evolutionary importance [1] and be related to various diseases such as mental retardation [2], neurological disorders [3,4], and cancers [5]. Although chromosomal abnormalities including CNVs were originally investigated using cytogenetic karyotype analyses such as chromosome banding analysis and fluorescence in situ hybridization (FISH), recent technologies such as pair-

\footnotetext{
* Correspondence: asako.koike.ea@hitachi.com

${ }^{1}$ Central Research Laboratory, Hitachi Ltd. Tokyo, Japan

Full list of author information is available at the end of the article
}

end sequencing [6-8], whole genome sequencing using massively parallel sequencers [9], and array-based approaches such as array comparative genomic hybridization (CGH) [10] and single nucleotide polymorphism (SNP) genotyping microarrays [11]) enabled us to obtain refined CNV structures with relatively high throughput. Particularly, SNP genotyping microarrays are becoming widely used for $\mathrm{CNV}$ identification since CNVs can be detected using the same microarrays used in the SNP-based case control study. The SNP microarray approaches, however, tend to lead to too many false negatives and positives and require sophisticated CNV detection methods/algorithms.

Many methods/algorithms have been proposed for detecting CNVs for array CGH and SNP array and are
C Biomed Central

(c) 2011 Koike et al; licensee BioMed Central Ltd. This is an Open Access article distributed under the terms of the Creative Commons Attribution License (http://creativecommons.org/licenses/by/2.0), which permits unrestricted use, distribution, and reproduction in any medium, provided the original work is properly cited. 
divided into several models such as smoothing methods, clustering methods, maximum likelihood procedures including Hidden Markov models (HMMs) and expectation-maximization (EM) algorithms. The simplest smoothing method is for smoothing $\log _{2}$ ratio (ratio is defined as the intensity of target of probe divided by that of reference probe) profiles using a moving average and detecting duplicated or deleted regions over the specified thresholds [12]. For more sophisticated smoothing methods, a quantile smoothing method based on L1 norm (the sum of absolute values) penalty minimization [13] and a wavelet de-noising method [14] have been proposed. For a clustering method, the cluster along with chromosomes (CLAC) method was developed, in which hierarchical clustering trees along each chromosome arm (or chromosome) are calculated and the 'interesting' clusters considering the false discovery rate (FDR) are selected [15]. Smoothing and clustering methods are effective in simulation data, but they do not achieve good enough CNV detection performance compared with other methods in array CGH experimental data [16]. To date, various maximum likelihoodrelated approaches have been proposed. Jong et al. introduced genetic local search algorithms (memetic algorithms) for maximizing the likelihood by considering the penalty function of breakpoints [17]. Picard et al. developed an adaptive method for estimating the penalty constant to avoid selecting too large a segmentation number for over fitting given data. In this method, the probe intensity profile ( $\log _{2}$ ratio) is supposed to be a Gaussian distribution, and the number of segments is estimated by maximizing the likelihood [18]. A circular binary segmentation (CBS) method was proposed by Venkatraman and Olshen [19], in which the average probe intensity is assumed to also have a Gaussian distribution. The likelihood ratio statistic for testing the null hypothesis, in which there is no change, and the alternative hypothesis, in which there is exactly one change at an unknown location, are introduced in this method. The test is done using a permutation test. If the null hypothesis is rejected, the hypothetical changepoints are adopted. The change-points are searched recursively using overlapping windows [19].

An HMM is a statistical model in which it is assumed that the system follows a Markov process [20-22]. In most HMM models for CNV detection methods, the probe intensity values or $\log R$ ratio (LRR, $\log _{2}\left(R_{\text {observed }} /\right.$ $\left.R_{\text {expected }}\right), R_{\text {expected }}$ is calculated from linear interpolation of canonical genotype clusters, $R$ is a sum of probe intensities) and B allele frequency (BAF, normalized measure of relative signal intensity ratio of the $B$ and $A$ alleles in the SNP array) or genotypes are assumed to be independent, and the copy number states of the probes are set to be hidden states with certain transition probabilities. By maximizing the likelihood of observed data (probe intensity, LRR and BAF, or genotypes), the copy number state of each probe is obtained.

Many studies have compared the performance of these methods or programs based mainly on simulation data, arrayCGH data [16] and Illumina SNP arrays [23]. However, Affymetrix SNP-array-based CNV detection requires much more robust algorithms than those using Illumina SNP arrays and array CGH due to the characteristics of the Affymetrix SNP arrays.

We assessed the following widely used methods/programs, the circular binary segmentation (CBS) method (implemented in DNAcopy [19], R package), Picard's adaptive method (CGHseg [18], R package), HMMs (Birdsuite 1.4 [20] and PennCNV [21]) on the basis of whether they accurately detect CNVs on Affymetrix data (Affymetrix 6.0). The first two methods/programs are known to be effective in detecting CNVs of array$\mathrm{CGH}$ experiments, but their performances in microarrays are yet unknown. QuantiSNP [22], which uses an objective Bayes HMM, showed the best detection performance with the simulation and Illumina SNP array data in a previous study [23]. We also tested QuantiSNP, but the parameter tunings for Affymetrix data were too difficult for us to achieve sufficient performance. Therefore, the results are not shown in the following results. The assessments are done using HapMap data and other experimental results. After that, CNVs of 180 healthy Japanese individuals were detected using the parameters that showed the best performance in the HapMap data. The characteristics of start and end regions of CNVs are also discussed. These results are registered in the $\mathrm{CNV}$ control database, which has been developed as part of the Japanese integrated database project.

\section{Methods}

\section{CNV detection methods}

We conducted comparative analyses on the CNV detection performance of five programs: DNAcopy [19] (R package) as a circular binary segmentation (CBS) method, CGHseg [18] ("tiling array" of R) as a Picard's adaptive method, Birdseye (part of Birdsuite) [20] and PennCNV [21] as HMMs, and Birdsuite (mainly consisting of Birdseye and the EM-based Canary).

There are two main subjects for CNV detection: estimation of the copy numbers and detection of accurate $\mathrm{CNV}$ boundaries from probe intensity data. In this study, duplications (gains) or deletions (losses) were assessed, but copy numbers were not assessed because there were not enough validation data to confirm them. In the programs for array $\mathrm{CGH}$, the $\log _{2}$ ratios of normalized intensities of target probes against control probes were used as input data, while programs for the 
SNP arrays, not only the $\log _{2}$ ratios or LRR but also allele frequencies or genotypes were also used in most cases. Since DNAcopy and CGHseg were developed for array $\mathrm{CGH}$, only $\log _{2}$ ratios were used as input data. During $\log _{2}$ ratio calculation, quantile-normalization was done for probe intensities and the average values of HapMap data (270 individuals) were used as reference probe intensity data.

Birdsuite consists of four programs (modules), "Canary", "Birdseed", "Birdseye", and "Fawkes," and determines CNVs using multiple individual data. In Canary, common CNVs are detected with EM algorithms using registered known common copy number data, while Birdseye detects novel CNVs by using the HMM with the Viterbi algorithm based on probe intensities and genotypes determined using the Birdseed program. Fawkes combines results of Canary, Birdseye, and Birdseed and assigns a comprehensive SNP genotype. Since we do not access the genotypes of CNVs, we used only Canary and Birdseye to obtain the following results.

In PennCNV, probe intensity data is converted into LRR and BAF, copy numbers are set as hidden states, and the emission probability of LRR and BAF are modeled. The hidden state for maximizing the likelihood of observed data (LRR and BAF) is obtained using the Viterbi algorithm.

\section{Data sets}

To compare the detection performance of these algorithms, we used Affymetrix Genome-wide Human SNP Array 6.0 (Affy 6.0) data of the HapMap data. The HapMap data were collected from 45 Japanese in Tokyo, Japan, 45 Han Chinese in Beijing, China, 90 Yoruba individuals (30 trios) in Ibadan, Nigeria, and 90 individuals from the US state of Utah with northern and western European ancestry (collected in 1980 by the Centre d'Etude du Polymorphisme Humain) whose CNVs were previously measured in other experiments and/or algorithms $[6-8,11,20,21,24-29]$. Furthermore, five sets of five Affy 6.0 microarrays of the same individuals (NA04626, NA01416, NA06061, NA10851, NA15510) were also used to investigate the reproducibility of the detection algorithm. These Affy 6.0 data were typed in Affymetrix and downloaded from the Affymetrix web site [30].

Furthermore, novel Affy 6.0 data of 180 healthy Japanese individuals, whose typing was carried out in our previous study [31], were used for detecting Japanese CNVs. (Hereinafter we call these data "original data.") As described in the previous paper [31], the study was approved by the research ethics committee of Central Research Laboratory, Hitachi Ltd. (permission number 128-2) and the Faculty of Medicine, The University of Tokyo (permission number 2583) and the informed consent was obtained from all participants.

\section{Database construction}

A CNV control database has been constructed as part of the integrated database project by the Ministry of Education, Culture, Sports, Science and Technology (MEXT) using mySQL. In the database, the start-end information of copy number segments, copy number regions which are clustered copy number segment information, and their frequencies were accumulated. Other information such as genes, exons, introns, and the CNVs of database of genomic variants (DGV [32]) data were also accumulated to enable users to easily interpret the meaning of detected $\mathrm{CNV}$ regions. The start and end positions of the CNVs were also stored on a distributed annotation system (DAS) with the GMOD Gbrowse-based [33] browser for calling up our data on other DAS servers, such as Ensemble, or vice versa; the data on another DAS are called up on our DAS to view various data simultaneously for interpretation.

\section{Results and discussion}

\section{Evaluation of CNV detection algorithms using trios data}

To evaluate the CNV detection performance of each method, the concordance of CNV detections was investigated using five sets of microarrays for the same individuals (five microarrays per individual $\times$ five individuals). Table 1 summarizes the average stability rates of CNVs at the segment level to measure $\mathrm{CNV}$ reproducibility using different DNA from the same individual. (Analyses were done by copy number segments if there are no explanations.) Copy number segments with overlap > $80 \%$ were regarded as concordant segments. Even if the overlap threshold was changed (20-80\%), this tendency was almost the same. In this stability rate, duplications and deletions were discriminated, but copy number differences within duplications or deletions were not distinguished. The CNV detection thresholds (parameters)

\section{Table 1 Average stability rate of CNVs using same} individual's data

\begin{tabular}{ll}
\hline Programs & Average of concordance rate \\
\hline PennCNV & $89.4 \%$ \\
Birdsuite:Birdseye/Canary & $91.1 / 94.6 \%$ \\
DNAcopy & $82.0 \%$ \\
CGHseg & $79.6 \%$ \\
\hline
\end{tabular}

Stability rate is the concordance rate of CNVs detected using different DNA samples from the same person. Segment overlap $>80 \%$ is regarded as concordant.

All versus all comparisons ( 5 microarray analysis $\times 5$ microarray analysis) were done.

PennCNV: Threshold of number of probes $>20$.

Birdseye (part of Birdsuite): Threshold of number of probes $>20$ and Lod score $>5$.

DNAcopy: Threshold of number of probes $>30$ and absolute avg. intensity of a segment $>0.35$.

CGHseg: Threshold of number of probes $>40$ and absolute avg. intensity of a segment $>0.35$. 
in each method were determined to maximize the stability rate. These thresholds show the best performance (minimum value) also in the Mendelian inconsistency ratio of trio data (Table 2). Furthermore, when other array data for the same individuals are used as reference samples instead of the average of HapMap data and these thresholds are applied, neither DNAcopy nor CGHseg show false positives (that is, CNVs are not detected).

The stability rate does not directly predict performance but indicates the stability of detection performance. Table 1 shows that PennCNV and Birdseye (part of Birdsuite) have the same level of detection stability, about $10 \%$ higher than those of DNA copy and CGHseg, while Canary (part of Birdsuite) achieves higher stability than others due to the utilization of pre-defined copy number regions.

Table 2 shows the rate of Mendelian inconsistencies of HapMap trio (90 individuals) data. The rate is calculated as the average number of an offspring's CNVs that are not detected in both parents divided by the number of each offspring's CNVs. Although a rate of Mendelian inconsistencies close to 0 does not directly mean high CNV detection performance, the rate of Mendelian inconsistencies much larger than 0 implies low $\mathrm{CNV}$ detection performance considering heritability because the frequency of copy number change is assumed to be in the range of $10^{-6}$ to $10^{-4}$ per gamete [24,34]. The rates of Mendelian inconsistencies of Canary (part of Birdsuite), and Birdseye (part of Birdsuite) in Table 2 are the lowest and second lowest, respectively; however, there may be influences of the parameters which are tuned using HapMap data [20] considering the low stability rate of Birdseye in Table 1 (that is, the square-root of the stability rate $<<1$-Mendelian inconsistency in Birdseye). For Canary, since only predefined regions are the targets of CNV detection, low Mendelian inconsistencies of Canary are reasonable. The low Mendelian inconsistency is a necessary but not sufficient condition for high accuracy for CNV detections. Mendelian inconsistencies of all methods, except Canary and Birdseye, are not sufficiently low, and DNAcopy shows the highest Mendelian inconsistencies, although the stability rate in Table 1 is the same level as CGHseg. The cause is not clear, but there might be CNV patterns that are

Table 2 The rate of Mendelian inconsistencies in offsprings' CNVs of HapMap trio data

\begin{tabular}{llll}
\hline Programs & Overlap $>\mathbf{0 \%}$ & $\mathbf{>} \mathbf{5 0 \%}$ & $\mathbf{>} \mathbf{8 0 \%}$ \\
\hline PennCNV & 0.190 & 0.192 & 0.197 \\
Birdsuite:Birdseye/Canary & $0.028 / 0.015$ & $0.030 / 0.015$ & $0.030 / 0.015$ \\
DNAcopy & 0.531 & 0.579 & 0.583 \\
CGHseg & 0.417 & 0.462 & 0.493 \\
\hline
\end{tabular}

difficult to detect with circular binary-segmentationbased detection for DNAcopy.

The similarity of CNVs detected using each program is measured as the sensitivity between programs in Table 3. Although PennCNV and Birdseye (part of Birdsuite) use a HMM, similarity between them is not so large in all (deletions + duplications) CNV data. The difference between them might be partly caused by parameter settings. Overall, deletions are higher than all (deletions + duplications) CNVs. DNAcopy shows a comparatively high similarity compared with all other programs considering the small number of CNVs detected by DNAcopy. The dependence of the similarity on CNV length was not clearly observed. In Figure 1 (a), the distributions of CNV length using each program/method are depicted, as discussed later.

Affymetrix data is known to be noisy for determining genotypes under some experimental conditions. When detecting CNVs, data noise is expected to be problematic. Supplemental Figures 1S-a (HapMap) and 2S-a (180 healthy Japanese individuals) [Additional file 1: Supplemental Figures $1 \mathrm{~S}$ and 2S] show the relationship between the standard deviation of $\log _{2}$ ratio in each array and $\mathrm{CNV}$ counts per individual, while supplemental Figures 1S-b (HapMap) and 2S-b (180 healthy Japanese individuals) [Additional file 1: Supplemental figures $1 \mathrm{~S}$ and $2 \mathrm{~S}$ ] show the relationships between the call rate (the percentage of SNPs whose genotypes are determined in the genotype calling process) and CNV counts per individual detected using each program. Figures 1Sa and 2S-a indicate that the CNV counts per individual drastically increase when the standard deviation of the $\log _{2}$ ratio of the array is high. Figures $1 \mathrm{~S}-\mathrm{b}$ and $2 \mathrm{~S}-\mathrm{b}$ indicate that Affymetrix arrays with low recall showed higher CNV counts. Since these tendencies were observed in all programs, arrays with large standard deviations or with low recall should be removed. In the analysis of original data in Section 'CNVs of original data of healthy Japanese', individuals with high CNV counts (15 individuals whose CNV number are PennCNV > 250, Birdseye (part of Birdsuite) > 400, Canary (part of Birdsuite) > 500, DNAcopy > 100, or CGHseg > 100) were removed.

\section{Evaluation of CNV detection algorithms by comparing other experimental results}

The CNVs of these HapMap individuals have also been investigated using various experimental approaches [6-8,11,24-29], and a comparison of these previous studies is summarized in Table 4. In Table 4, the HapMap individuals used in both this study and in each experiment are used to calculate sensitivity. The corresponding specificity is summarized in supplemental Table $1 \mathrm{~S}$ [Additional file 2: Supplemental Table 1S]. There are no 
Table 3 Similarity of CNVs detected between programs using HapMap data

\begin{tabular}{lllll}
\hline Programs & PennCNV & Birdseye (part of Birdsuite) Birdsuite & DNAcopy & CGHseg \\
\hline PennCNV & - & $55.1 / 62.2^{*}$ & $62.5 / 75.2$ & $49.2 / 63.5(51.5 / 65.3)$ \\
[12417/6895] & & $(56.4 / 61.9)$ & $(63.1 / 76.7)$ & \\
& $23.2 / 26.8$ & & \\
& & $(23.8 / 26.9)$ & $57.5 / 68.4$ & $40.7 / 52.4$ \\
Birdseye (part of Birdsuite) & $62.2 / 69.8$ & - & $(69.8 / 82.6)$ & $(49.1 / 61.6)$ \\
[14794/10102] & $(82.8 / 88.5)$ & & $68.8 / 73.9$ & $47.0 / 54.4$ \\
Birdsuite & $68.0 / 74.3$ & & $(84.5 / 89.4)$ & $(57.5 / 63.3)$ \\
[56476/44150] & $(90.3 / 93.3)$ & & - & $61.1 / 68.6$ \\
DNAcopy & $37.7 / 40.8$ & $29.6 / 31.8$ & $(62.1 / 68.6)$ \\
[6748/4849] & $(50.7 / 53.8)$ & $(38.0 / 40.4)$ & & - \\
& & $13.8 / 15.0$ & & \\
& & $(16.4 / 17.7)$ & $32.1 / 35.6$ & \\
CGHseg & $10.1 / 12.3$ & $(27.4 / 32.0)$ & \\
[3648/2401] & $(11.6 / 13.9)$ & & \\
& $(17.8 / 1 / 20.9)$ & $4.2 / 5.2$ & & \\
& & $(4.6 / 5.6)$ & & \\
\end{tabular}

Similarity: percentage of CNVs detected using the program in row, which is also detected using the program in column. That is, each program in row is regard as golden standard.

*Sensitivity with $80 \%$ overlap/5\%overlap of CNV segments.

(): represents deletion-only data.

[]: represents total number of CNVs/total number of CNV deletions.

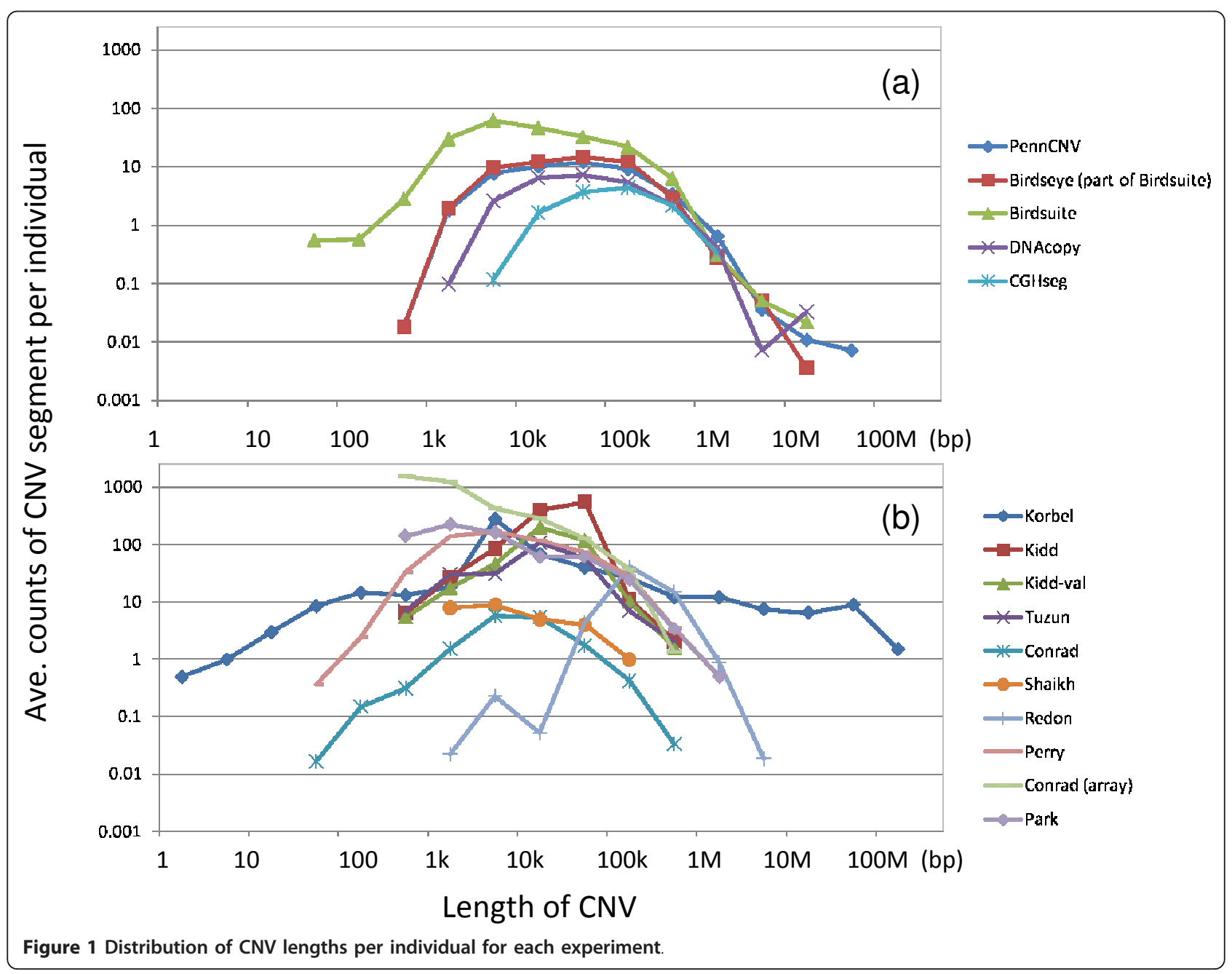


tendencies of longer CNVs being commonly detected in other experiments (data not shown). The overlap among these experiments' CNVs is low, as shown in supplemental Table 2S [Additional file 2: Supplemental Table $2 \mathrm{~S}]$. The inconsistent results of various experiments also make it difficult to validate $\mathrm{CNV}$ detection methods. These experimental data probably include many false negatives and some false positives. The sensitivities become slightly higher when the detection thresholds are changed. However, considering the CNVs' reproducibility (stability) for the same individual, these thresholds seem plausible. In most cases, the sensitivity and specificity are high in "deletion-only data". This is partly because some of the experimental results do not distinguish "insertion" and "duplication". When the results of Birdseye (part of Birdsuite) and Birdsuite are compared, the sensitivities are increased but the specificities are largely decreased; because of that, the specificity of Canary (part of Birdsuite) is low. Since these other experiments are expected to include false negatives, low specificity may not directly mean low accuracy of the program. Given that the CNV detection's stability of the same individuals is high and the Mendelian inconsistency ratio is comparatively low in PennCNV, Birdseye (part of Birdsuite), and Birdsuite (mainly consisting of Birdseye and Canary), they are expected to show higher sensitivity and specificity than those of other programs. It should be noted that the sensitivities of Birdseye (part of Birdsuite), Birdsuite, and PennCNV in Table 4 are higher than those of DNAcopy and CGHseg in Conrad [28] and Park's [29] results, which are expected to be more reliable than earlier data such as Redon [11] and
Korbel [6] when their estimated false discovery rate (Conrad et al. 15\% [28]) and Mendelian inconsistencies (Park et al. 2.6\% [29]) are considered, although the sensitivities of all programs are quite low in some experiments. Table 1S, however, implies that there are no striking differences among specificities of these CNV detection programs. This indicates that detection programs are expected to have many false negatives and a smaller number of false positives.

The distributions of CNV length are summarized in Figure 1. As shown in Figure 1 (a), the difference of distributions of CNV length between each program is not so large and especially the distribution of CNV length between Birdseye (part of Birdsuite) and PennCNV, which both use HMM, is similar. When Figures 1 (a) and (b) are compared, the lengths of the newly detected CNVs are longer than other experimental results and the total number of detected CNVs are less than others, which are partly caused of used thresholds for detecting CNVs. Shorter CNVs are also detected using these programs before applying thresholds. The distributions of $\mathrm{CNV}$ lengths are quite different depending on the CNV experimental method, even if the same individual is used, as shown in Figure 1 (b). Although a gain or loss $<1 \mathrm{~kb}$ is not regarded as a CNV but as a deletion or insertion in the DGV criteria [32], all gain and loss data were used as CNVs in this study. When sequencing techniques were used, small gain and loss could be detected, as shown in this figure. On the other hand, when SNP array or array CGH was used, only long CNVs could be detected due to the sparseness of the probe density on a genome.

Table 4 Sensitivity of each detection program when other experimental result is regarded as golden standard

\begin{tabular}{|c|c|c|c|c|c|c|c|c|c|c|}
\hline Programs & $\begin{array}{l}\text { Korbel [6] } \\
\text { (PE } \\
\text { sequencing) }\end{array}$ & $\begin{array}{l}\text { Kidd [7] (PE } \\
\text { sequencing) }\end{array}$ & $\begin{array}{l}\text { Kidd [7] } \\
\text {-validation }\end{array}$ & $\begin{array}{l}\text { Tuzun [8] } \\
\text { (PE } \\
\text { sequencing) }\end{array}$ & $\begin{array}{l}\text { Conrad [25] } \\
* * \\
\text { (Mendelian } \\
\text { consistency) }\end{array}$ & $\begin{array}{l}\text { Shaikh } \\
{[26] * *} \\
\text { (Illumine } \\
\text { Data) }\end{array}$ & $\begin{array}{l}\text { Redon } \\
\text { [11] } \\
\text { (Tiling } \\
\text { array) }\end{array}$ & $\begin{array}{l}\text { Perry } \\
\text { [27] } \\
\text { (array } \\
\text { CGH) }\end{array}$ & $\begin{array}{l}\text { Conrad } \\
\text { [28] } \\
\text { (Tiling } \\
\text { array) }\end{array}$ & $\begin{array}{l}\text { Park [29] (array- } \\
\text { CGH and } \\
\text { massively paralle } \\
\text { sequencing) }\end{array}$ \\
\hline PennCNV & $\begin{array}{l}2.2^{*} \\
(2.1)\end{array}$ & $\begin{array}{l}0.5 \\
(0.7)\end{array}$ & $\begin{array}{l}1.2 \\
(1.5)\end{array}$ & $\begin{array}{l}5.2 \\
(7.8)\end{array}$ & 23.7 & $\begin{array}{l}37.0 \\
{[37.1]}\end{array}$ & $\begin{array}{l}1.2 \\
(1.1)\end{array}$ & $\begin{array}{l}18.1 \\
(22.3)\end{array}$ & $\begin{array}{l}26.2 \\
(24.5)\end{array}$ & $\begin{array}{l}27.0 \\
(33.0)\end{array}$ \\
\hline $\begin{array}{l}\text { Birdseye } \\
\text { (part of } \\
\text { Birdsuite) }\end{array}$ & $\begin{array}{l}2.4 \\
(2.4)\end{array}$ & $\begin{array}{l}0.6 \\
(1.0)\end{array}$ & $\begin{array}{l}1.4 \\
(2.1)\end{array}$ & $\begin{array}{l}7.7 \\
(13.9)\end{array}$ & 30.0 & $\begin{array}{l}40.7 \\
{[44.4]}\end{array}$ & $\begin{array}{l}0.8 \\
(0.9)\end{array}$ & $\begin{array}{l}13.4 \\
(14.0)\end{array}$ & $\begin{array}{l}23.5 \\
(24.1)\end{array}$ & $\begin{array}{l}27.9 \\
(36.9)\end{array}$ \\
\hline Birdsuite & $\begin{array}{l}5.5 \\
(6.1)\end{array}$ & $\begin{array}{l}2.0 \\
(4.3)\end{array}$ & $\begin{array}{l}5.5 \\
(8.8)\end{array}$ & $\begin{array}{l}17.2 \\
(24.8)\end{array}$ & 46.1 & $\begin{array}{l}51.9 \\
{[70.4]}\end{array}$ & $\begin{array}{l}2.2 \\
(3.2)\end{array}$ & $\begin{array}{l}25.6 \\
(30.0)\end{array}$ & $\begin{array}{l}35.4 \\
(35.9)\end{array}$ & $\begin{array}{l}42.9 \\
(56.2)\end{array}$ \\
\hline DNAcopy & $\begin{array}{l}1.7 \\
(1.6)\end{array}$ & $\begin{array}{l}0.4 \\
(0.9)\end{array}$ & $\begin{array}{l}1.1 \\
(1.9)\end{array}$ & $\begin{array}{l}5.2 \\
(9.0)\end{array}$ & 20.4 & $\begin{array}{l}44.4 \\
{[48.1]}\end{array}$ & $\begin{array}{l}0.7 \\
(1.2)\end{array}$ & $\begin{array}{l}9.9 \\
(11.8)\end{array}$ & $\begin{array}{l}15.3 \\
(17.9)\end{array}$ & $\begin{array}{l}19.1 \\
(28.7)\end{array}$ \\
\hline CGHseg & $\begin{array}{l}0.5 \\
(0.3)\end{array}$ & $\begin{array}{l}0.1 \\
(0.3)\end{array}$ & $\begin{array}{l}0.3 \\
(0.6)\end{array}$ & $\begin{array}{l}1.5 \\
(2.7)\end{array}$ & 18.2 & $\begin{array}{l}29.6 \\
{[29.6]}\end{array}$ & $\begin{array}{l}0.4 \\
(0.7)\end{array}$ & $\begin{array}{l}7.0 \\
(6.4)\end{array}$ & $\begin{array}{l}7.7 \\
(9.3)\end{array}$ & $\begin{array}{l}9.6 \\
(12.6)\end{array}$ \\
\hline
\end{tabular}

*Sensitivity of all data, () represents deletion-only data.

Sensitivity: Common data between "other experimental results" and "each CNV detection program's results" divided by "other experimental results".

Commonly utilized individuals between this study and other experiment are used for calculating sensitivity and specificity.

CNV segments with overlap $>80 \%$ are regarded as commonly detected segments.

Kidd-validation: Kidd results that are also detected using other experimental methods in the paper ${ }^{7}$.

**Conrad and Shaikh data include only deletion data.

[] represents ratios with overlapping ratio $>30 \%$ and CNV length $>10 \mathrm{~kb}$. 
The results of the section 'Evaluation of CNV detection algorithms using trios data' and 'Evaluation of CNV detection algorithms by comparing other experimental results' indicate that Hidden Markov based programs PennCNV and Birdseye (part of Birdsuite), or Birdsuite (mainly consisting of Birdseye and EM-based Canary) are superior to others when the high $\mathrm{CNV}$ reproducible (stability) rates of the same individuals and the low Mendelian inconsistencies are taken into account. For measuring sensitivities with other experimental results, Birdsuite is the best. However, overlapping rates with other experimental results suggest that there remain many false negatives and some false positives, although other experimental results are also expected to contain many false positives and negatives.

\section{CNVs of original data of healthy Japanese Overlap between DGV, HapMap, and original data}

$\mathrm{CNVs}$ of the original 180 healthy Japanese individuals are detected by these five programs using parameters that achieved the best performance in HapMap data in Section 'Evaluation of CNV detection algorithms using trios data'. The similarity of CNVs detected using each program is summarized as the sensitivity in supplemental Table 3S [Additional file 2: Supplemental Table 3S]. Table 5 lists the overlap ratios (specificity) of HapMap data and original data with Conrad's data (JPT +CHB 90 individuals) [28] and Park's data (30 Asian individuals) [29] and summarizes the influence of commonality among individuals. Unlike Table 4, the overlap ratio (specificity) of Table 5 was calculated not by individual level, but by whole level (pooled CNVs). Compared to the CNVs detected in the HapMap data, the overlap ratios of the original data with Conrad's data and Park's data are low in all programs except Birdsuite. In every case, Birdsuite, especially the Birdseye module, shows high overlap ratio. When regions commonly detected using at least two programs are selected, the overlap ratio increases except for the results from the Birdseye (part of Birdsuite), as shown in Table 5. Therefore, it is expected that commonly detected CNVs using two programs are reliable CNV regions. When the number of commonly detected individuals increases, the overlap ratio also increases as shown in Table 5, suggesting that the repeatedly detected regions are plausible common CNVs.

The numbers of commonly detected CNVs with HapMap data and original data of healthy Japanese are summarized in supplemental Table 4S [Additional file 2: Supplemental Table 4S]. Since the overlap between original data and HapMap data should be JPT/CHB > CEU $>$ YRI, considering the genetic distance of ethnics, the tendency $\mathrm{JPT} / \mathrm{CHB}>\mathrm{CEU}>\mathrm{YRI}$ in any program is reasonable in Table $4 \mathrm{~S}$.

\section{Characteristics of CNV regions}

As interpretations of origin of copy number variations, two mechanisms have been proposed [35]. One is the non-allelic homologous recombination (NAHR, ectopic HR) mediated mechanism and the other is a microhomology-based mechanism. NAHR requires long repeated sequences (up to $300 \mathrm{bp}$ in human [36]) in the start and end regions of CNVs, while microhomology requires only $5-15$ bp homology sequences. NAHR is expected to occur by unequal crossing-over and break-induced replication (BIR). Single-strand annealing (SSA) is also a deletion mechanism [35]. In SSA, the complementary single-stranded sequences of the 5 '-end of a doublestrand break are annealed, and the regions between the two complementary sequences are deleted.

Table 5 Overlap ratio of original data and HapMap data with Park and Conrad's data

\begin{tabular}{|c|c|c|c|c|c|}
\hline Programs & $\begin{array}{l}\text { Average of } \\
\text { overlap ratio } \\
\text { of HapMap } \\
\text { with Conrad's } \\
\text { data [28] and } \\
\text { Park's data } \\
\text { [29] }\end{array}$ & $\begin{array}{l}\text { Average of } \\
\text { overlap ratio of } \\
\text { original data } \\
\text { with Conrad's } \\
\text { data [28] and } \\
\text { Park's data [29] }\end{array}$ & $\begin{array}{l}\text { Average of overlap ratio } \\
\text { of original data of } \\
\text { commonly detected } \\
\text { regions at least two } \\
\text { programs, with Conrad's } \\
\text { data [28] and Park's } \\
\text { data [29] }\end{array}$ & $\begin{array}{l}\text { Average of overlap ratio } \\
\text { of original data of } \\
\text { commonly detected } \\
\text { regions in more than } \\
\text { two individuals, with } \\
\text { Conrad's data [28] and } \\
\text { Park's data [29] }\end{array}$ & $\begin{array}{l}\text { Average of overlap ratio } \\
\text { of original data of } \\
\text { commonly detected } \\
\text { regions in more than } \\
\text { nine individuals with } \\
\text { Conrad's data [28] and } \\
\text { Park's data [29] }\end{array}$ \\
\hline PennCNV [8986/5619] & $\begin{array}{l}52.2 / 53.2 \% \%^{* \$} \\
44.6 / 50.8 \%^{\$ \$}\end{array}$ & $\begin{array}{l}35.8 / 39.7 \% \\
32.1 / 39.0 \%\end{array}$ & $\begin{array}{l}42.5 / 45.2 \% \\
42.7 / 48.2 \%\end{array}$ & $\begin{array}{l}57.8 / 58.6 \% \\
58.6 / 63.9 \%\end{array}$ & $\begin{array}{l}61.8 / 62.3 \% \\
62.9 / 68.0 \%\end{array}$ \\
\hline $\begin{array}{l}\text { Birdseye (part of Birdsuite) } \\
\text { [6959/5063] }\end{array}$ & $\begin{array}{l}68.7 / 69.6 \% \\
62.9 / 65.6 \%\end{array}$ & $\begin{array}{l}71.0 / 71.6 \% \\
81 . / 83.2 \%\end{array}$ & $\begin{array}{l}62.1 / 63.1 \% \\
71.4 / 74.6 \%\end{array}$ & $\begin{array}{l}75.6 / 75.8 \% \\
86.7 / 88.5 \%\end{array}$ & $\begin{array}{l}79.4 / 79.5 \% \\
91.6 / 92.9 \%\end{array}$ \\
\hline Birdsuite [31852/25794] & $\begin{array}{l}66.3 / 66.6 \% \\
53.4 / 56.5 \%\end{array}$ & $\begin{array}{l}65.0 / 65.3 \% \\
63.8 / 66.8 \%\end{array}$ & $\begin{array}{l}65.2 / 65.9 \% \\
73.9 / 77.1 \%\end{array}$ & $\begin{array}{l}67.9 / 67.9 \% \\
67.1 / 70.1 \%\end{array}$ & $\begin{array}{l}70.6 / 70.6 \% \\
69.7 / 72 / 7 \%\end{array}$ \\
\hline DNAcopy [10243/4436] & $\begin{array}{l}63.2 / 64.2 \% \\
49.6 / 54.1 \%\end{array}$ & $\begin{array}{l}24.2 / 27.8 \% \\
20.0 / 25.6 \%\end{array}$ & $\begin{array}{l}33.8 / 36.6 \% \\
33.4 / 38.6 \%\end{array}$ & $\begin{array}{l}66.4 / 66.8 \% \\
61.9 / 66.4 \%\end{array}$ & $\begin{array}{l}72.5 / 72.5 \% \\
67.6 / 71.1 \%\end{array}$ \\
\hline CGHseg [4043/1966] & $\begin{array}{l}54.7 / 56.6 \% \\
38.9 / 45.4 \%\end{array}$ & $\begin{array}{l}31.1 / 38.8 \% \\
21.9 / 31.7 \%\end{array}$ & $\begin{array}{l}38.1 / 41.6 \% \\
33.7 / 40.4 \%\end{array}$ & $\begin{array}{l}68.0 / 69.6 \% \\
56.3 / 63.3 \%\end{array}$ & $\begin{array}{l}74.5 / 75.5 \% \\
60.8 / 65.5 \%\end{array}$ \\
\hline
\end{tabular}

*ratio of $80 \%<$ CNV overlap segments/ratio of $5 \%<$ CNV overlap segments.

\$:Comparison with Conrad's data, \$ Comparison with Park's data.

[]: represents total number of CNVs/total number of CNV deletions in original data. 
The ratios of the CNV start and end regions where segmental repeats are included, are listed in Table 6. The segmental repeats annotated by UCSC [37] were used. Segmental repeats are sequences that have sequence similarity with another genomic location ( $>1 \mathrm{~kb}$ and $>90 \%$ sequence similarity). Although the threshold of repeat length is not imposed in Table 6, the tendencies are not changed even if the threshold is set at $300 \mathrm{bp}$. The $\pm 1 \mathrm{kbp}$ in Table 6 indicates the regions $\pm 1 \mathrm{kbp}$ of the $\mathrm{CNV}$ start and end region, that is, $-1 \mathrm{k}$ bp to $+1 \mathrm{k}$ bp of the start region and $-1 \mathrm{kbp}$ to $+1 \mathrm{kbp}$ for the end region are used. These comparatively long start and end regions are used because the probes in an array are sparse and both experimental and computational errors are expected in the start and end regions of detected CNVs. Even when the regions are narrowed to $\pm 500 \mathrm{bp}$, the tendency of the results is not changed, as shown in Table 6. Common 1 represents the CNV regions detected using at least two programs, while Common 2 represents the $\mathrm{CNV}$ regions detected in at least five individuals by using PennCNV and at least one other program. The enrichment of segmental repeats in CNV regions was reported in previous studies [11,38]. Table 6 shows that the ratios of CNVs including segmental repeats in both start and end regions are higher than those of randomly selected regions. The differences between Common 1 and Common 2 are not large. Table 6 also lists the ratios of CNVs including interspersed repeats such as long and short interspersed nuclear elements (LINE and SINE, respectively) and long terminal repeat (LTR) in the start and end regions of CNVs. The repeats identified in UCSC [37] using Repeat Masker 3.2.7 were used. The CNV ratios, including repeats in both the start and end regions in Common1 and Common2, are significantly higher than those of randomly selected regions in any interspersed repeat kind except "only SINE". The ratios of Common1 and Common2 are higher than those of randomly selected regions also in "LTR", but the difference is small. Furthermore, the ratios of Common2 are higher than those of Common 1 in most cases of any interspersed repeat kind except "only SINE".

In all detection programs, with an increasing number of commonly detected individuals, the CNV ratios containing interspersed repeats or segmental repeats also increased as shown in supplemental Table $5 \mathrm{~S}$ [Additional file 2: Supplemental Table 5S]. (It should be noted that there are no obvious relationships between these ratios and the stability rate or the Mendelian inconsistency ratio of each program.) When the ratios of CNVs including "segmental repeats or interspersed repeats" in start and end regions of CNVs are calculated, the results are higher than "segmental repeats" only or "interspersed repeats (ALL)" only as shown in Table 6. About $40 \%$ of segmental repeats detected around CNVs include interspersed repeats. Of these segmental repeats, LINE and LTR are about $50 \%$ and $15 \%$, respectively. Since LINE, SINE, and LTR account for about 52\%, $14 \%, 23 \%$ of all interspersed repeats in the human genome (hg18), respectively, there are no LINE biases in the segmental repeats around CNVs. The enrichment of interspersed repeats and segmental repeats is also observed in previous experimental data as shown in supplemental Table 6S [Additional file 2: Supplemental Table 6S] with a few exceptions.

These results indicate that not only segmental repeats but also interspersed repeat regions, especially "LINEs", have an important role in the formation of CNVs, at least in frequently observed copy number variations, although there is a possibility that interspersed repeats promote segmental repeats. Although both SINE and LINE elements have been reported to contribute structural

Table 6 The segmental repeats and interspersed repeats-included percentage of start and end regions of CNV

\begin{tabular}{|c|c|c|c|c|c|c|}
\hline Programs & Segmental repeats & ALL* & Only SINE & Only LINE & Only LTR & Segmental repeat + interspersed repeats (ALL*) \\
\hline $\begin{array}{l}\text { Random* } \\
\pm 500 \mathrm{bp}\end{array}$ & 2.8 & $17.3^{*}$ & 0.0 & 7.8 & 1.6 & 19.6 \\
\hline $\begin{array}{l}\text { Random } \\
\pm 1 \mathrm{kbp}\end{array}$ & 2.9 & 21.7 & 0.4 & 10.6 & 1.9 & 24.1 \\
\hline $\begin{array}{l}\text { CNVCommon } 1 \\
\pm 500 \mathrm{bp}\end{array}$ & 34.0 & 24.8 & 0.0 & 12.4 & 2.3 & 53.9 \\
\hline $\begin{array}{l}\text { CNVCommon } 1 \\
\pm 1 \mathrm{kbp}\end{array}$ & 34.6 & 39.5 & 1.3 & 21.9 & 5.6 & 62.7 \\
\hline $\begin{array}{l}\text { CNVCommon2 } \\
\pm 500 \mathrm{bp}\end{array}$ & 32.2 & 29.1 & 0.0 & 11.6 & 2.5 & 53.7 \\
\hline $\begin{array}{l}\text { CNVCommon2 } \\
\pm 1 \mathrm{kbp}\end{array}$ & 33.1 & 43.8 & 0.0 & 25.8 & 5.0 & 65.3 \\
\hline
\end{tabular}

$\mathrm{ALL}^{*}$ means all interspersed repeats of repeatmasker 3.2.7.

CNVcommon 1: 315 CNV regions detected by at least two programs and more than once.

CNVcommon2: 121 CNV regions detected by PennCNV more than four times (that is, at least five individuals) and at least one other program. Random*: 1000 randomly extracted regions whose lengths are close to extracted CNV lengths. 
Table 7 Average conservation score of start and end of CNV regions

\begin{tabular}{lll}
\hline Regions & $\begin{array}{l}\text { Average } \\
\text { conservation score } \\
\text { in start regions }\end{array}$ & $\begin{array}{l}\text { Average } \\
\text { conservation score } \\
\text { in end regions }\end{array}$ \\
\hline Random $\pm 500 \mathrm{bp}$ & 62.7 & 67.8 \\
Random $\pm 800 \mathrm{bp}$ & 60.9 & 77.0 \\
Random $\pm 1 \mathrm{kbp}$ & 69.1 & 76.0 \\
CNVCommon1 $\pm 500 \mathrm{bp}$ & 38.2 & 51.1 \\
CNVCommon1 $\pm 800 \mathrm{bp}$ & 42.5 & 55.6 \\
CNVCommon1 $\pm 1 \mathrm{kbp}$ & 47.9 & 62.3 \\
CNVCommon2 $\pm 500 \mathrm{bp}$ & 32.9 & 47.6 \\
CNVCommon2 $\pm 800 \mathrm{bp}$ & 35.7 & 50.8 \\
CNVCommon2 $\pm 1 \mathrm{kbp}$ & 39.4 & 57.0 \\
\hline
\end{tabular}

CNVcommon1: 315 CNV regions detected by at least two programs and more than once.

CNVcommon2: 121 CNV regions detected by PennCNV more than four times and at least one other program.

Scored sum value of phastCons44wayPrimates (UCSC) is used as Conservation score. variations [39], our results and those of others [11] do not support a contribution of SINE to formation of CNVs.

When the CNV ratios including simple repeats and mobile elements (provided in UCSC) in both the start and end regions are compared, there are no differences among Common1, Common2, and random values. The recombination rate of these regions $( \pm 500 \mathrm{bp}$ ) calculated using the Marshfield average does not show statistically meaningful differences among random values $(=1.17)$ and CNV common1 ( = 1.14) and common2 $(=1.18)$.

The expected length, 5-15 bp, of microhomology is too short to use array-based $\mathrm{CNV}$ detection methods. In many CNV start and end regions, microhomologous sequences were found but there were almost no statistical significances (for example, 4 bases $^{\wedge} 5 \mathrm{bp}$ is at most 1024) when the start and end positions of CNVs were ambiguous due to sparseness of probe positions. Conrad et al. [40] have investigated breakpoints of CNV

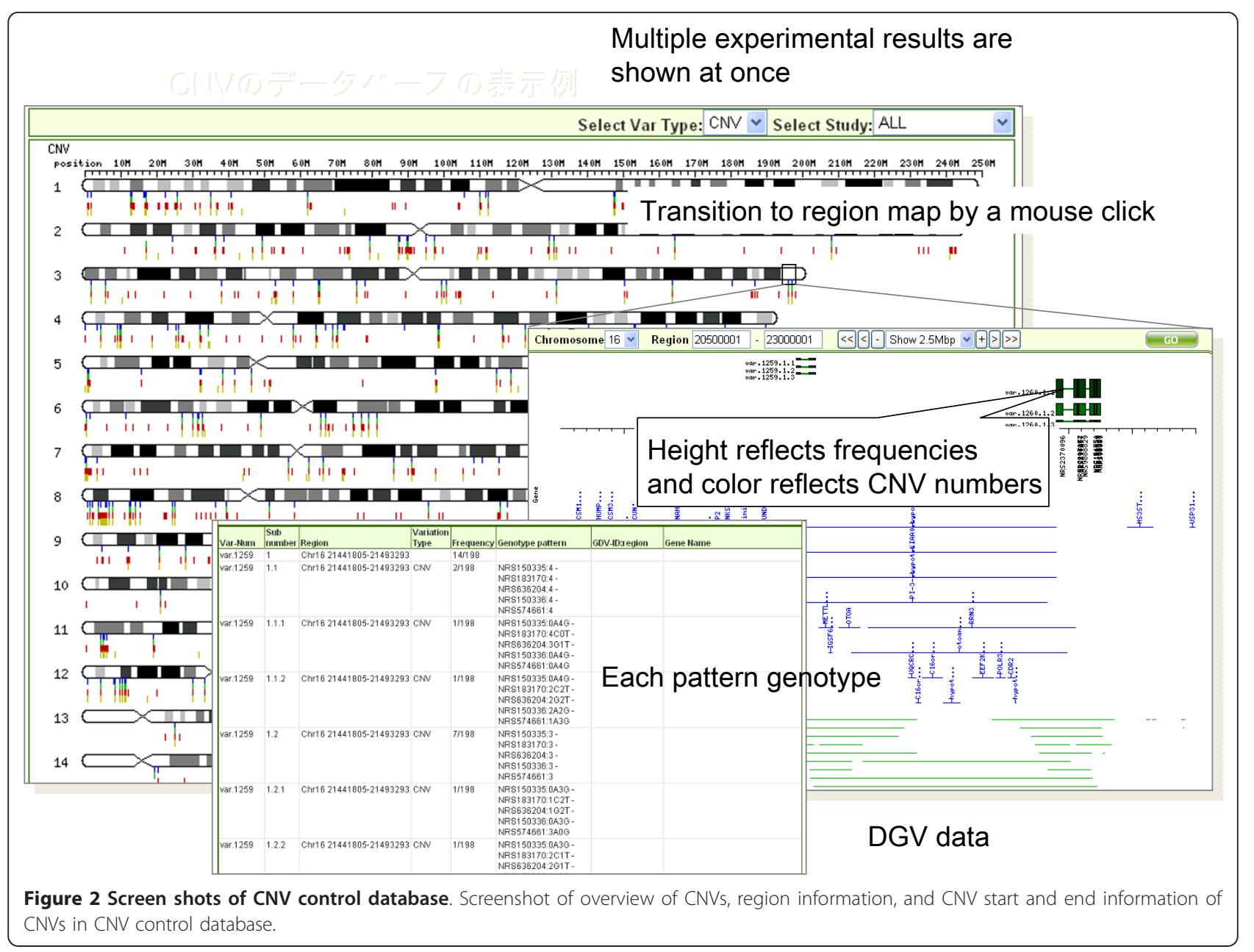


deletions by massive parallel sequencer and found $70 \%$ of them have microhomology. Sequencing of these regions is necessary to confirm their importance.

Table 7 compares the conservation scores from primates (UCSC phastCons44wayPrimates [37]). This table indicates that the start and end regions of $\mathrm{CNVs}$ are lower than random values, and CNV Common2 shows smaller conservation scores than those of CNV Common1. In all detection methods, as the number of commonly detected individuals increased, the average conservation score of the CNV start and end regions decreased (data not shown). When the number of CpG islands is counted in the CNV start and end regions, it is slightly smaller than those in random regions $(\mathrm{P}=$ 0.034). This seems reasonable because $\mathrm{CpG}$ islands are sparse in repeat regions.

The above-mentioned tendencies in the start and end regions of $\mathrm{CNVs}$ were also observed in our $\mathrm{CNV}$ results of HapMap data, and differences between CNV duplications and deletions were not observed. It should be noted that although the overlapping ratio between our $\mathrm{CNV}$ results of HapMap data and other experimental data are low, most of them have the similar tendency that both segmental repeats and interspersed repeats (especially LINE) are enriched in CNV start and end regions, and conservation scores based on primates are lower in these regions than in randomly selected regions.

\section{CNV database construction}

In the Japanese Integrated Database Project of MEXT, our organization (Univ. of Tokyo, Univ. of Tokai, and Hitachi, Ltd.) has constructed and maintained a public repository for the genome-wide association study (GWAS) database for continuous and intensive management of GWAS data and to facilitate data-sharing among researchers [41]. A CNV control database has been constructed as a part of the GWAS database.

Since the start and end positions are slightly different between individuals, this makes it difficult to understand common CNV structures. Accordingly, CNVs with start and end positions located within 20 probes are regarded as the same $\mathrm{CNV}$ regions by clustering CNVs of all individuals. Screenshots of the CNV control database are shown in Figures 2 and 3. As shown in Figure 2, interfaces for the overview of CNVs on whole genomes and for region information are provided. Results both with and without clustering can be depicted, as shown in Figure 3. The abovementioned original data are stored in this database.

In this system, bulk CNV data are freely available, but a simple application is required for accessing $\mathrm{CNV}$ data

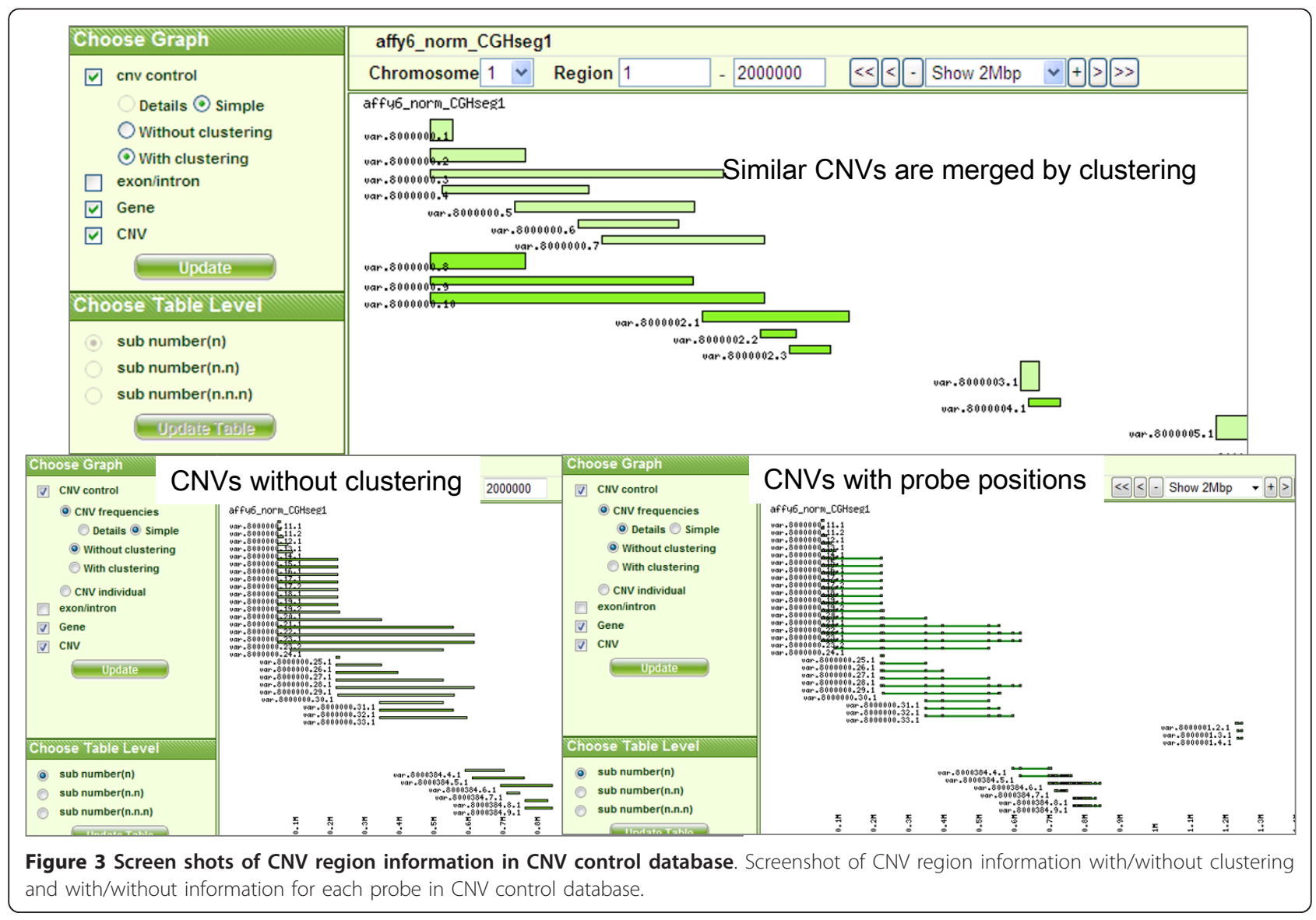


at the individual level. To use raw data for CNVs, researchers must submit an application describing the research purpose in detail to the database access committee. Access is determined at the data sharing review board. The details of the data sharing policy are summarized in http://gwas.lifesciencedb.jp/gwasdb/db_policy_en.html.

The CNV control database is accessible at http://gwas. lifesciencedb.jp/cgi-bin/cnvdb/cnv_top.cgi.

\section{Conclusions}

In this study, we evaluated five widely used CNV detection programs, Birdsuite, Birdseye (part of Birdsuite), PennCNV, CGHseg, and DNAcopy from the viewpoint of performance on the Affymetrix platform using HapMap data and other experimental data. Our results indicate that hidden Markov based programs PennCNV and Birdseye (part of Birdsuite), or Birdsuite are superior to others when the high CNV detection stability (reproducibility) rates of the same individuals and the low Mendelian inconsistencies are considered. For measuring the sensitivity of other experimental results, Birdsuite shows the best performance. However, the low overlapping rates with other experimental results imply that there remain many false negatives and some false positives, although other experimental results also contain many false positives and negatives.

The analysis of start and end regions of CNVs in the data for healthy Japanese and the HapMap data showed that both segmental repeats and interspersed repeats are enriched in CNV start and end regions, suggesting that not only segmental repeats but also interspersed repeats, especially LINE, are deeply involved in CNV formation, particularly in common CNV formations, although the previous studies mainly focused on segmental repeats $[9,11]$. There are CNVs without segmental repeats or interspersed repeats. They might contain microhomologies or other characteristics, the resolution of SNP array seems too coarse to analyze microhomologies. Other sequence level technologies will be required for further detailed analysis.

\section{Additional material}

Additional file 1: Supplementary Figures. Figure is (a) Relationship between standard deviation of probe intensity $\log _{2}$ ratio of each microarray and number of CNV segments per individual in HapMap data. (b) Relationship between call rate (the percentage of probes with genotypes determined in the genotype calling process) and number of CNV segments per individual in HapMap data. Figure $2 S$ (a) Relationship between standard deviation of probe intensity $\log _{2}$ ratio of each microarray and number of CNV segments per individual in original data. (b) Relationship between call rate and number of CNV segments per individual in original data.

Additional file 2: Supplementary Tables.

\section{Acknowledgements}

This work was supported by the contract research fund "Integrated Database Project" from the Ministry of Education, Culture, Sports, Science, and Technology of Japan. We thank anonymous reviewers for helpful comments.

\section{Author details}

${ }^{1}$ Central Research Laboratory, Hitachi Ltd. Tokyo, Japan. ${ }^{2}$ Department of Human Genetics, Graduate School of Medicine, University of Tokyo, Tokyo, Japan.

\section{Authors' contributions}

KT participated in the conception and design of the study and interpreted the results. NN and DY designed and coordinated the experiment. AK participated in the conception and design of the study, carried out the analysis, and wrote the manuscript. All authors reviewed analysis results and read and approved the final manuscript.

Received: 29 August 2010 Accepted: 7 March 2011

Published: 7 March 2011

\section{References}

1. Dumas L, Kim YH, Karimpour-Fard A, Cox M, Hopkins J, Pollack JR, et al: Gene copy number variation spanning 60 million years of human and primate evolution. Genome Res 2007, 17:1266-1277.

2. Friedman JM, Baross A, Delaney AD, Ally A, Arbour L, Armstrong $L$, et al: Oligonucleotide microarray analysis of genomic imbalance in children with mental retardation. Am J Hum Genet 2006, 79:500-513.

3. Glessner JT, Reilly MP, Kim CE, Takahashi N, Albano A, Hou C, et al: Strong synaptic transmission impact by copy number variations in schizophrenia. Proc Natl Acad Sci USA 2010, 107:10584-10589.

4. Sundaram SK, Huq AM, Wilson BJ, Chugani HT: Tourette syndrome is associated with recurrent exonic copy number variants. Neurology 2010, 74:1583-1590.

5. Shlien A, Malkin D: Copy number variations and cancer. Genome Med 2009, 1:62.

6. Korbel JO, Urban AE, Affourtit JP, Godwin B, Grubert F, Simons JF, et al: Paired-end mapping reveals extensive structural variation in the human genome. Science 2007, 318:420-426.

7. Kidd JM, Cooper GM, Donahue WF, Hayden HS, Sampas N, Graves T, et al: Mapping and sequencing of structural variation from eight human genomes. Nature 2008, 453:56-64.

8. Tuzun E, Sharp AJ, Bailey JA, Kaul R, Morrison VA, Pertz LM, et al: Fine-scale structural variation of the human genome. Nat Genet 2005, 37:727-732.

9. Alkan C, Kidd JM, Marques-Bonet T, Aksay G, Antonacci F, Hormozdiari F, et al: Personalized copy number and segmental duplication maps using next-generation sequencing. Nat Genet 2009, 41:1061-1067.

10. Perry GH, Yang F, Marques-Bonet T, Murphy C, Fitzgerald T, Lee AS, et al: Copy number variation and evolution in humans and chimpanzees. Genome Res 2008, 18:1698-1710.

11. Redon R, Ishikawa S, Fitch KR, Feuk L, Perry GH, Andrews TD, et al: Global variation in copy number in the human genome. Nature 2006, 444:444-454.

12. Pollack JR, Sørlie T, Perou CM, Rees CA, Jeffrey SS, Lonning PE, et al: Microarray analysis reveals a major direct role of DNA copy number alteration in the transcriptional program of human breast tumors. Proc Natl Acad Sci USA 2002, 99:12963-12968.

13. Eilers PH, de Menezes RX: Quantile smoothing of array CGH data. Bioinformatics 2005, 21:1146-1153.

14. Hsu L, Self SG, Grove D, Randolph T, Wang K, Delrow JJ, et al: Denoising array-based comparative genomic hybridization data using wavelets. Biostatistics 2005, 6:211-226.

15. Wang P, Kim Y, Pollack J, Narasimhan B, Tibshirani R: A method for calling gains and losses in array CGH data. Biostatistics 2005, 6:45-58.

16. Lai WR, Johnson MD, Kucherlapati R, Park PJ: Comparative analysis of algorithms for identifying amplifications and deletions in array CGH data. Bioinformatics 2005, 21:3763-3770.

17. Jong K, Marchiori E, van der Vaart A, Ylstra B, Weiss M, Meijer G: Chromosomal Breakpoint Detection in Human Cancer. Lecture Notes in Computer Science 2003, 2611:107-116. 
18. Picard F, Robin S, Lavielle M, Vaisse C, Daudin JJ: A statistical approach for array CGH data analysis. BMC Bioinformatics 2005, 11(6):27.

19. Venkatraman $E S$, Olshen $A B: A$ faster circular binary segmentation algorithm for the analysis of array CGH data. Bioinformatics 2007, 23:657-663.

20. Korn JM, Kuruvilla FG, McCarroll SA, Wysoker A, Nemesh J, Cawley S, et al: Integrated genotype calling and association analysis of SNPs., common copy number polymorphisms and rare CNVs. Nat Genet. 2008 40:1253-1260.

21. Wang K, Li M, Hadley D, Liu R, Glessner J, Grant SF, et al: PennCNV: an integrated hidden Markov model designed for high-resolution copy number variation detection in whole-genome SNP genotyping data. Genome Res 2007, 17:1665-1674.

22. Colella S, Yau C, Taylor JM, Mirza G, Butler H, Clouston P, et al: QuantiSNP: an Objective Bayes Hidden-Markov Model to detect and accurately map copy number variation using SNP genotyping data. Nucleic Acids Res 2007, 35:2013-2025.

23. Dellinger AE, Saw SM, Goh LK, Seielstad M, Young TL, Li YJ: Comparative analyses of seven algorithms for copy number variant identification from single nucleotide polymorphism arrays. Nucleic Acids Research 2010, 38:e105

24. Turner DJ, Miretti M, Rajan D, Fiegler H, Carter NP, Blayney ML, et al: Germline rates of de novo meiotic deletions and duplications causing several genomic disorders. Nat Genet 2008, 40:90-95.

25. Conrad DF, Andrews TD, Carter NP, Hurles ME, Pritchard JK: A highresolution survey of deletion polymorphism in the human genome. Nat Genet 2006, 38:75-81.

26. Shaikh TH, Gai X, Perin JC, Glessner JT, Xie H, Murphy K, et al: Highresolution mapping and analysis of copy number variations in the human genome: a data resource for clinical and research applications. Genome Res 2009, 19:1682-1690.

27. Perry GH, Ben-Dor A, Tsalenko A, Sampas N, Rodriguez-Revenga L, Tran CW, et al: The fine-scale and complex architecture of human copy-number variation. Am J Hum Genet 2008, 82:685-695.

28. Conrad DF, Pinto D, Redon R, Feuk L, Gokcumen O, Zhang Y, et al: Origins and functional impact of copy number variation in the human genome. Nature 2010, 464(7289):704-12.

29. Park H, Kim Jl, Ju YS, Gokcumen O, Mills RE, Kim S, et al: Discovery of common Asian copy number variants using integrated high-resolution array CGH and massively parallel DNA sequencing. Nat Genet 2010, 42(5):400-5

30. The Affymetrix Web Site. [http://www.affymetrix.com/jp/index.affx].

31. Nishida N, Koike A, Tajima A, Ogasawara Y, Ishibashi Y, Uehara Y, et al: Evaluating the performance of Affymetrix SNP Array 6.0 platform with 400 Japanese individuals. BMC Genomics 2008, 22(9):431.

32. The Database of Genomic Variants (GDV). [http://projects.tcag.ca/ variation/]

33. The Generic Model Organism Database (GMOD) project. [http://gmod org/wiki/Main_Page].

34. van Ommen GJ: Frequency of new copy number variation in humans. Nat Genet 2005, 37:333-334.

35. Hastings PJ, Lupski JR, Rosenberg SM, Ira G: Mechanisms of change in gene copy number. Nat Rev Genet 2009, 10:551-564.

36. Reiter LT, Hastings PJ, Nelis E, De Jonghe P, Van Broeckhoven C, Lupski JR: Human meiotic recombination products revealed by sequencing a hotspot for homologous strand exchange in multiple HNPP deletion patients. Am J Hum Genet 1998, 62:1023-1033.

37. UCSC genome browser. [http://hgdownload.cse.ucsc.edu/goldenPath/ hg18/database/]

38. Sharp AJ, Locke DP, McGrath SD, Cheng Z, Bailey JA, Vallente RU, et al: Segmental duplications and copy-number variation in the human genome. Am J Hum Genet 2005, 77:78-88.

39. Iskow RC, McCabe MT, Mills RE, Torene S, Pittard WS, Neuwald AF, et at: Natural mutagenesis of human genomes by endogenous retrotransposons. Cell 2010, 25:1253-1261.

40. Conrad DF, Bird C, Blackburne B, Lindsay S, Mamanova L, Lee C, Turner DJ, Hurles ME: Mutation spectrum revealed by breakpoint sequencing of human germline CNVs. Nat Genetic 2010, 42:38-391.
41. Koike A, Nishida N, Inoue I, Tsuji S, Tokunaga K: Genome-wide association database developed in the Japanese Integrated Database Project. J Hum Genet 2009, 54:543-546.

doi:10.1186/1471-2156-12-29

Cite this article as: Koike et al:: Comparative analysis of copy number variation detection methods and database construction. BMC Genetics 2011 12:29.

\section{Submit your next manuscript to BioMed Central and take full advantage of:}

- Convenient online submission

- Thorough peer review

- No space constraints or color figure charges

- Immediate publication on acceptance

- Inclusion in PubMed, CAS, Scopus and Google Scholar

- Research which is freely available for redistribution

Submit your manuscript at www.biomedcentral.com/submit
Ciomed Central 